\title{
Gender Differences of Achilles tendon Cross-sectional Area during Loading
}

\section{(ㄷ) (1) $\circledast \odot$}

Authors

Konstantina Intziegianni, Michael Cassel, Gerrit Hain, Frank Mayer

\begin{abstract}
Affiliations
Sports Medicine and Sports Orthopaedics, University

Outpatient Clinic Potsdam, Potsdam, Germany
\end{abstract}

Key words

Achilles tendon, reliability, cross-sectional area, compliance, strain, gender

received 20.04 .2017

revised 24.05 .2017

accepted 05.06 .2017

\section{Bibliography}

DOI https://doi.org/10.1055/s-0043-113814

Sports Medicine International Open 2017; 1: E135-E140

(c) Georg Thieme Verlag KG Stuttgart · New York

ISSN 2367-1890

\author{
Correspondence \\ Dr. Konstantina Intziegianni, Ph.D. \\ University Outpatient Clinic Potsdam \\ Sports Medicine and Sports Orthopaedics \\ University of Potsdam \\ Am Neuen Palais 10, Haus 12 \\ 14469, Potsdam \\ Germany \\ Tel.: +49/331/9774 916, Fax: +49/331/9771 296 \\ intziegi@uni-potsdam.de
}

\begin{abstract}
The Achilles tendon (AT) is larger and stiffer in males compared to females. AT stiffness is determined by length differences during loading. However, as some collagen fibres run transversely, changes in cross-sectional area (CSA) are also expected. The study investigates the gender differences of AT-CSA during maximal voluntary isometric contraction (MVIC).

Fifteen males and fifteen females were positioned prone on the isokinetic dynamometer with knee extended and ankle flexed $90^{\circ}$. AT-CSA [ $\mathrm{mm}^{2}$ ] from rest to MVIC during plantar flexion was sonographically assessed. AT-CSA maximal deformation [ $\mathrm{mm}^{2}$ ] was subtracted by $\mathrm{CSA}_{\mathrm{MVIC}}-\mathrm{CSA}_{\text {rest }}$. AT-CSA compliance $\left[\mathrm{mm}^{2} /\right.$ $\mathrm{Nm}$ ] and strain [\%] were calculated by dividing the CSA deformation $\left[\mathrm{mm}^{2}\right]$ by peak torque $[\mathrm{Nm}]$ and CSA at rest $\left[\mathrm{mm}^{2}\right]$, respectively. Gender differences were assessed by an independent sample t-test with Bonferroni correction $(\alpha=0.01)$.

AT-CSA dimensions at rest $(p=0.001)$ and contraction $(p=0.001)$ as well peak torque $(p=0.001)$ were statistically significant higher in males $\left(54.4 \pm 5.1 \mathrm{~mm}^{2}, 53.7 \pm 5.1 \mathrm{~mm}^{2}\right.$, $120.1 \pm 26.8 \mathrm{Nm})$ compared to females $\left(46.2 \pm 7.0 \mathrm{~mm}^{2}\right.$, $\left.43.4 \pm 6.9 \mathrm{~mm}^{2}, 86.9 \pm 21.6 \mathrm{Nm}\right)$. AT-CSA deformation $(p=0.000)$ strain $(p=0.000)$ and compliance $(p=0.000)$ were found to be statistically significant higher in females $\left(-2.8 \pm 0.9 \mathrm{~mm}^{2}\right.$, $\left.-6.2 \pm 2.0 \%,-0.033 \pm 0.018 \mathrm{~mm}^{2} / \mathrm{Nm}\right)$ compared to males $\left(-0.8 \pm 1.8 \mathrm{~mm}^{2},-1.4 \pm 3.3 \%,-0.007 \pm 0.008 \mathrm{~mm}^{2} / \mathrm{Nm}\right)$.

During loading, the AT also deforms at the transverse level by reducing its CSA. CSA reduction was higher in females, indicating also higher CSA compliance compared to males. Higher CSA compliance might indicate higher adaptability towards loading and might be discussed as a protective factor.
\end{abstract}

\section{Introduction}

The Achilles tendon (AT) is the strongest tendon in the human body and is exposed to high forces during daily activities and athletics $[8,11,12]$. In consequence, the AT is particularly susceptible to both chronic and acute injuries such as tendinopathy or tendon ruptures, which are often assumed to be related to the repetitive high loads [20,23]. Those types of tendon pathologies have been shown to be gender-specific with the ratio of AT rupture in men to women to be 5:1 [37] . Among the factors that might be responsible, tendon stiffness is of particular interest [10]. Stiffness refers to the degree of resistance offered by the tissues in response to stretching during loading. In the case of the AT, the displacement of the medial gastrocnemius myotendinous junction during loading is most often used to determine its stiffness [31].

Previous studies have shown that males have stiffer tendons compared to females $[4,5,9,22,29,40]$, which could partly explain the higher rate of injuries in males $[6,15,23,37]$. These gender-related differences found in tendon properties could be attributed to the increased body mass and force production capabilities observed in males [28] and increased estrogen levels found in females 
$[3,26]$. Increased body mass requires weight-bearing tendons to tolerate higher loads whereas increased muscle mass, which is directly associated with force production capabilities, requires tendons to transfer high loads. Those requirements lead to specific adaptations of the tendon by increasing its CSA dimensions and/or its material properties, thus leading to increased stiffness [28, 39].

Quantification of tendon structural dimensions and especially that of CSA is of importance in the research field as it is a variable needed for the calculation of tendon stress. The stress $\left(\mathrm{N} / \mathrm{mm}^{2}\right)$ imposed on the tendon during muscle contraction is calculated as the force transmitted to the tendon divided by its CSA dimensions at rest [25]. From an injury standpoint, larger CSA dimensions in asymptomatic tendons are advantageous in reducing the stress imposed on the tendon for a given load [25]. Strain [\%] is another important variable in understanding tendon behavior describing the relationship between tendon length deformation during contraction and its resting length dimensions [14]. According to invitro models, strain levels above $8 \%$ causes tendon microruptures and at $12 \%$ a complete tendon ruptured [38]. However, those values were recently underestimated during functional tasks with strains reaching levels of up to $16 \%$ during a single leg jump without causing tendon injury [18].

When subjected to tensile load imposed by muscle contraction, tendons undergo a longitudinal deformation by changing their length. However, as some collagen fibres run also transversely $[19,35]$, CSA deformation is also expected. Currently, no study has investigated the degree of deformation in CSA under contraction. Therefore the aim of the present study was to investigate the gender-specific AT-CSA deformation from rest to maximal voluntary isometric contraction. As females demonstrate a higher compliance compared to males at the longitudinal level by differences in length, it is hypothesised that females will also demonstrate a higher compliance at the transverse level by a higher AT-CSA deformation during contractions compared to males.

\section{Material and Methods}

\section{Participants}

Thirty healthy recreationally active participants ( 15 males and 15 females) volunteered in the present study ( $\triangleright$ Table 1 ). Sample size was determined via power analysis done on preliminary data. An effect size of 2.00 was calculated for CSA deformation based on an $\alpha$-level of 0.05 and power values of 0.80 , requiring a minimum number of 6 participants. A standardized clinical examination, including ultrasonography, was performed by a sports orthopaedic physician. Participants were excluded if they reported any acute or chronic musculoskeletal injury of the lower limb and/or signs of tendinopathy on ultrasound imaging [7]. All participants completed an informed consent form.

\section{Study design}

A cross-sectional design was used in the present study. The measurements and their further analysis were supported by a single investigator with three years of experience. The study was approved by the local ethics committee and met the ethical standards of the International Journal of Sports Medicine [13].

\section{Region of interest}

In the present study, the dominant limb was identified by asking the participants to electively use either the left or right foot to kick a ball, the elected side was considered as the non-dominant [30]. Participants were positioned prone on the examination table with hip and knee extended and ankle flexed at $90^{\circ}$. A diagnostic ultrasound device (Vivid q; GE Healthcare, Tirat Carmel, Israel) with a 7.5 $\mathrm{MHz}$ continuous linear ultrasound array (4-13 MHz) was used. Presets were standardized at a frequency of $13 \mathrm{MHz}$ and a depth of $3 \mathrm{~cm}$. The ultrasound video clips were recorded at a rate of 40 frames per second. The AT distal insertion was sonographically detected by use of metal fine wires placed between the skin and the transducer, overlaying the corresponding structure and providing an acoustic shadow visual to the ultrasound. This method was used to accurately mark the location of the AT distal insertion on the skin $[16,17]$. The region of interest was defined at a distance of $6 \mathrm{~cm}$ from the AT distal insertion by the use of a measuring tape and marked on the skin [17]. The region of $6 \mathrm{~cm}$ from the distal insertion is the most reliable site to measure AT-CSA at rest [17]. Furthermore, injuries, e. g. tendinopathy and ruptures, most often occurred at this so- called "mid-portion" [24].

\section{Ultrasonographic assessment of AT-CSA}

Participants were positioned prone on the isokinetic dynamometer (Con-trex MJ, Physiomed, Germany) with the hip and knee extended and ankle flexed at $90^{\circ}$ ( $\triangleright$ Fig. 1). The axis of rotation was carefully aligned with the lateral malleolus. The foot was strapped securely to the footplate by use of Velcro straps. AT-CSA was initially assessed during rest. The probe was place on the defined point on the AT by the investigator and three image scans were taken. For warm-up and familiarisation with the measurement procedure, a standardized warm-up protocol was chosen consisting of three submaximal and two maximal isometric plantar flexion contractions of $5 \mathrm{~s}$, with $1 \mathrm{~min}$ rest in between $[1,17,18]$. After these practice trials, participants performed 3 maximal isometric plantar flexion contractions of $3 \mathrm{~s}$ with $1 \mathrm{~min}$ rest. AT-CSA during the contractions was recorded simultaneously with the investigator holding the probe on the defined location ( $\triangleright$ Fig. 1 ).

\section{Reliability of the methodology used}

In the context of a pilot study, the reliability of the ultrasonographic methodology and image analysis used in the present study for the assessment of AT-CSA was also evaluated at rest and under contraction. Inter-rater reliability of AT-CSA was assessed in a randomised order within the same day by use of the same equipment by two investigators (experienced investigator (three years) and inexperienced investigator (one month focused training prior the study)). Intra-rater reliability was assessed by the experienced in-

- Table 1 Anthropometric characteristics of participants.

\begin{tabular}{|l|c|c|c|}
\hline Variables & Females $(\mathbf{N}=\mathbf{1 5})$ & Males $(\mathbf{N}=\mathbf{1 5})$ & P Value \\
\hline Age $[\mathrm{yr}]$ & $28 \pm 3$ & $30 \pm 4$ & 0.063 \\
\hline Height $[\mathrm{cm}]$ & $167 \pm 5$ & $182 \pm 7$ & $0.000^{*}$ \\
\hline Weight $[\mathrm{kg}]$ & $62 \pm 8$ & $81 \pm 7$ & $0.000^{*}$ \\
\hline \multicolumn{3}{|l|}{ Values are means \pm SD and depict group average of data. } \\
* Significant group differences (P $\leq 0.01)$
\end{tabular}


vestigator within an interval of one week. Reliability was assessed by Intraclass Correlation Coefficient (ICC, 2.1) with a $95 \%$ confidence interval ( $\mathrm{Cl}: 95 \%)$. An ICC value $\leq 0.50$ was considered low, 0.50 to 0.75 was considered moderate, $\geq 0.75$ was considered good and $\geq 0.90$ was considered excellent [32]. The agreement between the measurements was verified qualitatively using Bland-Altman analysis (Bias \pm Limits of Agreements, [LoA]) and was calculated by the following equation:

\section{Bias $\pm 1.96 \times S D$}

Variability was calculated as the absolute differences between the two investigators (inter) and between the two measurements (intra) divided by their average and expressed as percentage [\%]. Additionally, to provide an estimate of the precision of measurement, the standard error of measurement (SEM) was calculated by the following equation:

\section{$\mathrm{SEM}=\mathrm{SD} \times \sqrt{1-\mathrm{ICC}}$}

The reliability values for both intra- and inter-rater are presented in $>$ Table 2. Analysis demonstrated a good to excellent reliability with low levels of variability for CSA assessment at rest and contraction both intra- as well as inter-rater.

\section{Data analysis}

The ultrasound images and video clips of AT-CSA were stored digitally as DICOM files and processed on a PC using a public domain $\mathrm{NIH}$ image program (image], (http://rsb.info.nih.gov/nih-image/)). The freehand selection tool was used to outline the tendon and measure the CSA both at rest and during MVIC ( $\triangleright$ Fig. 2). AT-CSA during the MVIC was outlined at each maximal deformation by manually tracking the CSA from rest to MVIC. In order to decrease the variability within subjects, each image and video clip was digi- tized three times and the average was taken. The analysis was performed in a blinded procedure in order to minimize a possible bias in the results from the investigator preference or expectations. All images and video clips were stored under a four-digit random number that was assigned prior to testing and stored in an identification file. As a consequence, the investigator was blinded to the participants and measurement day. After finalizing the analysis of the data, the results were assigned to the corresponding participants.

\section{AT-CSA deformation}

To describe the change in CSA from rest to MVIC, the following equation was used:

\section{CSA Deformation $=$ CSA MVIC $\left[\mathrm{mm}^{2}\right]-$ CSA Rest $\left[\mathrm{mm}^{2}\right]$}

Traditionally tendon strain has been used to describe the longitudinal strain (length change (elongation)/resting length) along the axis of the tendon. In the present study, this calculation was modified and transferred to the transverse level taking into account the change in CSA during the contraction by its resting dimensions.

\section{AT-CSA strain}

To describe the change in CSA deformation in relation to its CSA dimensions at rest, CSA tendon strain was calculated by the following equation:

$$
\text { CSA strain }=\frac{\text { CSA Deformation }\left[\mathrm{mm}^{2}\right]}{\text { CSA Rest }\left[\mathrm{mm}^{2}\right]} \times 100
$$

Tendon compliance is usually calculated by dividing the tendon elongation by tendon force. Both tendon force and torque are indirect ways of expressing the forces acting on the tendon. In the present study a modification of this equation was made and trans-
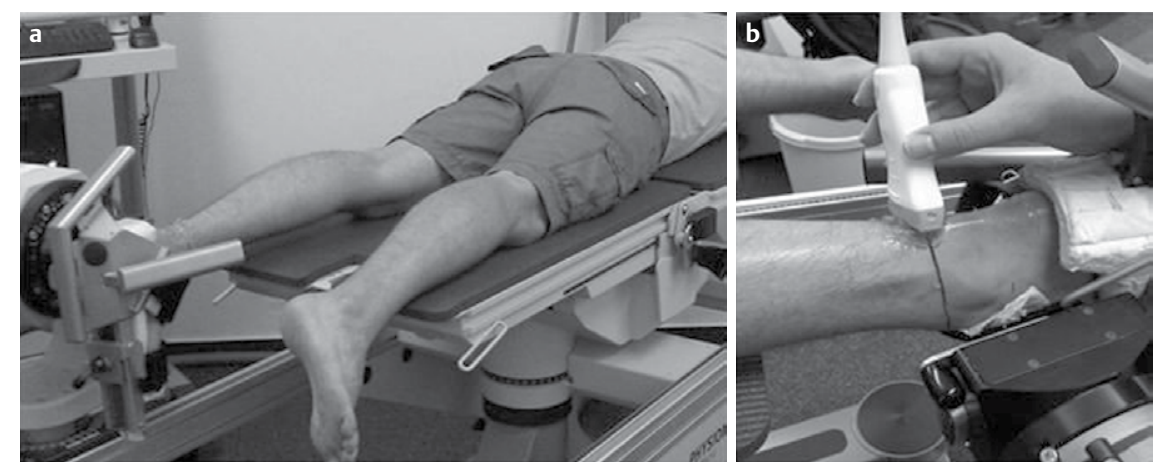

Fig. 1 Participants position on the isokinetic dynamometer and probe placement by the investigator over the region of interest.

- Table 2 Reliability of assessing Achilles tendon cross-sectional area (CSA) at rest and maximal voluntary isometric contraction (MVIC).

\begin{tabular}{|c|c|c|c|c|c|c|c|c|}
\hline & \multicolumn{4}{|c|}{ Intra-rater } & \multicolumn{4}{|c|}{ Inter-rater } \\
\hline $\mathrm{N}=42$ (24 females; 18 males) & $\operatorname{ICC}[2,1]$ & TRV [\%] & Bias \pm LoA $\left[\mathrm{mm}^{2}\right]$ & SEM $\left[\mathrm{mm}^{2}\right]$ & $\operatorname{ICC}[2,1]$ & IRV [\%] & Bias \pm LoA $\left[\mathrm{mm}^{2}\right]$ & SEM $\left[\mathrm{mm}^{2}\right]$ \\
\hline CSA at rest $\left[\mathrm{mm}^{2}\right]$ & 0.94 & $4 \pm 4$ & $-1 \pm 5$ & 2 & 0.84 & $8 \pm 4$ & $3 \pm 7$ & 3 \\
\hline $\mathrm{CSA}$ at $\mathrm{MVIC}\left[\mathrm{mm}^{2}\right]$ & 0.95 & $4 \pm 3$ & $-1 \pm 2$ & 2 & 0.87 & $6 \pm 4$ & $2 \pm 7$ & 3 \\
\hline
\end{tabular}

Measures of reliability: ICC = Intraclass correlation coefficient, TRV/IRV = test-retest/inter-rater variability, Bias \pm LoA $=95 \%$ limits of agreement,

SEM $=$ standard error of measurement 

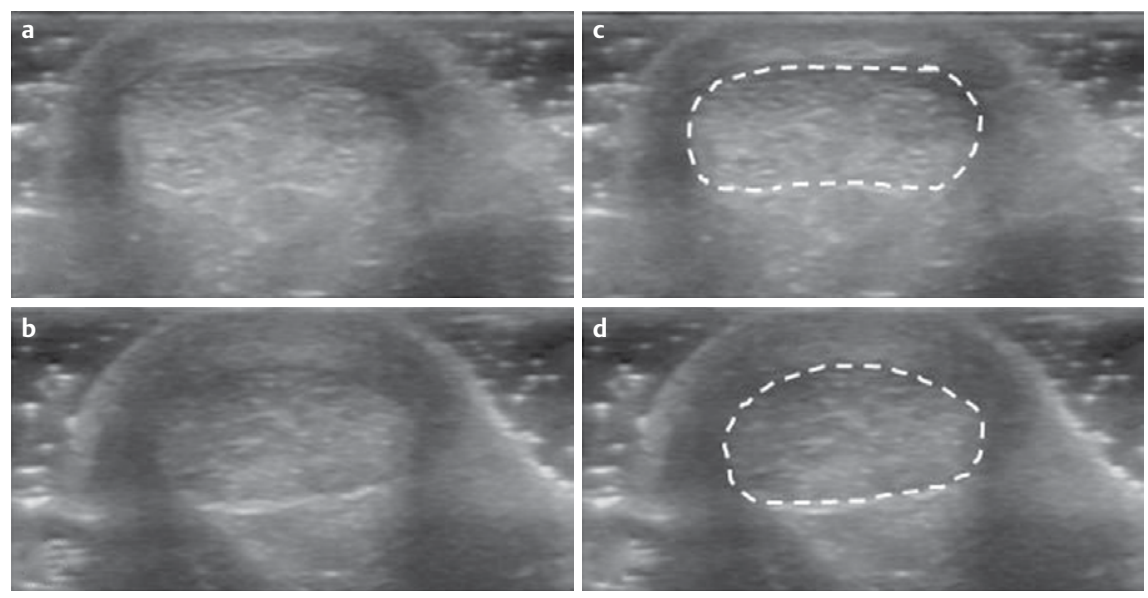

- Fig. 2 Achilles tendon Cross-sectional area (CSA) at rest (a, c) and under maximal voluntary isometric contraction (b, d). The white dotted lines outline the tendinous structure defining the CSA.

- Table 3 Achilles tendon cross-sectional area (CSA) properties and peak torque between males and females.

\begin{tabular}{|l|c|c|c|}
\hline Variables & Males & Females & PValue \\
\hline CSA at rest $\left[\mathrm{mm}^{2}\right]$ & $54.4 \pm 5.1$ & $46.2 \pm 7.0$ & $0.001^{*}$ \\
\hline CSA at contraction $\left[\mathrm{mm}^{2}\right]$ & $53.7 \pm 5.1$ & $43.4 \pm 6.9$ & $0.000^{*}$ \\
\hline CSA deformation $\left[\mathrm{mm}^{2}\right]$ & $-0.9 \pm 1.8$ & $-2.8 \pm 0.9$ & $0.000^{*}$ \\
\hline Peak torque[Nm] & $120.1 \pm 26.8$ & $86.9 \pm 21.6$ & $0.001^{*}$ \\
\hline CSA strain [\%] & $-1.4 \pm 3.3$ & $-6.2 \pm 2.0$ & $0.000^{*}$ \\
\hline CSA compliance $\left[\mathrm{mm}^{2} / \mathrm{Nm}\right]$ & $-0.007 \pm 0.008$ & $-0.033 \pm 0.018$ & $0.000^{*}$ \\
\hline $\begin{array}{l}\text { Values are means } \pm S \mathrm{SD} \text { and depict group average of data. }{ }^{*} \text { Significant } \\
\text { group differences }(\mathrm{P} \leq 0.01)\end{array}$ \\
\hline
\end{tabular}

ferred to the transverse level taking into account the deformation of the CSA divided by the peak torque.

\section{AT-CSA compliance}

To describe the deformation of the CSA in relation to the peak torque, the following equation was used:

CSA compliance $=\frac{\text { CSA Deformation }\left[\mathrm{mm}^{2}\right]}{\text { Peak Torque }[\mathrm{Nm}]}$

\section{Statistical analysis}

All statistical calculations were performed using SPSS (SPSS Statistics 22, IBM, USA). Data were initially analysed descriptively (mean \pm SD). Gender differences for AT-CSA dimensions at rest and MVIC, deformation, torque, strain and compliance were compared using an independent sample t-test followed by Bonferroni correction for multiplicity $(\alpha=0.01)$.

\section{Results}

The average values (mean \pm SD) for anthropometric characteristics, variables measured and statistical test used, are given in $>$ Tables 1 and 3. Males demonstrated a statistically significant larger AT-CSA dimensions both at rest (range $=$ males: 47.9 to $66.3 \mathrm{~mm}^{2}$, females: 33.2 to $55.6 \mathrm{~mm}^{2}, \mathrm{p}=0.001$ ) as well as during MVIC (range = males: 47.2 to $65.0 \mathrm{~mm}^{2}$, females: 30.4 to $\left.53.3 \mathrm{~mm}^{2}, \mathrm{p}=0.000\right)$ and a statistically significant higher peak torque (range $=$ males: 74.0 to $159.0 \mathrm{Nm}$, females: 54.0 to $119.0 \mathrm{Nm}, \mathrm{p}=0.001$ ). On the other hand, females demonstrated a statistically significant higher ATCSA deformation (range $=$ males: -4.3 to $2.6 \mathrm{~mm}^{2}$, females: -4.4 to $-1.2 \mathrm{~mm}^{2}, \mathrm{p}=0.000$ ), strain (range $=$ males: -8.1 to $5.0 \%$, females: -8.7 to $-2.2 \%, \mathrm{p}=0.000$ ) and compliance (range $=$ males: -0.044 to $0.030 \mathrm{~mm}^{2} / \mathrm{Nm}$, females: -0.050 to $-0.022 \mathrm{~mm}^{2} / \mathrm{Nm}$, $\mathrm{p}=0.000)$.

\section{Discussion}

The present study aimed to investigate the gender differences of AT-CSA under maximal isometric contraction. The results indicate that the AT also deforms at its transverse level by reducing its CSA. Females demonstrated a significantly higher CSA deformation under MVIC, indicating also a more transversely compliant tendon compared to males. Thus, these findings confirm the hypothesis that females will demonstrate higher AT-CSA deformation and compliance during contractions compared to males.

Previously, studies investigating the gender-related differences in tendon properties have led to the conclusion of higher tendon elongation and smaller structures in females exhibiting lower tendon stiffness compared to males [4, 5, 9, 22, 29, 40]. The present study adds that gender-related differences in CSA during loading can also be observed. Females demonstrated higher CSA deformation with lower forces indicating a more compliant tendon. As the ratio of injuries is higher in males compared to females[15, 37], higher compliance might allow a better adaptation towards loading.

In the present study, males demonstrated significantly larger CSA dimensions both at rest and during contraction compared to females. These findings are in line with previous studies reporting that males have larger tendon dimensions [4, 5, 9, 22, 29, 40]. These increased CSA dimensions found in males could be an adap- 
tation of AT towards increased force generation capacity and higher body mass compared to females. As the stiffness of its structures depends on its dimensions, it could be speculated that the higher compliance in CSA found in females is attributable to their smaller structures. However, this relationship is quite ambiguous [27] as there are studies which have shown that stiffness in tendons is independent of its dimensions [18, 34, 36]. Hence other factors such as tendon micro-structure could be assumed to also play a role, e. g. increased fibril diameter, fibril packing [33], collagen crosslinking [2] and reduced collagen crimping [21].

Calculations of tendon mechanical and material properties are essential for understanding mechanisms that enable to optimize the functional behavior of the muscle-tendon complex [1]. Stress $\left(\mathrm{N} / \mathrm{mm}^{2}\right)$ is a variable which is used in order to determine the material properties of the tendon, the so-called Young's modulus (stress/strain). Traditionally, stress is calculated by taking CSA dimensions at rest [25]. However, as those dimensions are changing under loading with females demonstrating $7 \%$ and males $2 \%$ of CSA reduction also the estimated stress values are deem to change leading to underestimation between $2 \%-7 \%$ of this calculated variable. This finding leads to critical considerations of tendon stress calculations and future studies should address this issue as this might result in a false estimations of tendon young's modulus.

To better understand the changes of CSA during loading in relation to its dimensions, an attempt was made to calculate its strain $\left(\mathrm{CSA}_{\text {deformation }} / \mathrm{CSA}_{\text {rest }}\right)$. The result revealed that females have a significantly higher CSA strain compared to males. Higher tendon strains are thought to cause ruptures in the longitudinal level of tendons when strain (elongation/length) reaches a level between $8 \%-12 \%$ [38]. However those values were based on in-vitro models and do not represent how tendons respond to loading under physiological loads [18]. The findings of the present study demonstrated that during isometric contractions, CSA strain can reach levels of up to $-9 \%$ without causing any injuries. However, to be able to classify CSA strain, more investigation is also needed in pathological tendons in order to understand the possible implications.

Although the methodology was reliable in the assessment of CSA both at rest and during contraction, the definition of the region of interest should be critically discussed. In the present study, the region of interest was defined at $6 \mathrm{~cm}$ from the distal attachment. Since CSA dimensions are not the same throughout its length, this region was specifically selected because it was shown to have the highest reproducibility [17]. As males in the present study were significantly taller than females, it could be argued that the region of interest in males was defined more distally. However, a recent study by Intziegianni et al. showed that taller participants do not necessarily have a longer Achilles tendon [18]. Thus, for a more accurate comparison between participants, a percentage of distance rather than a standardized point should be selected.

The findings of this study provide important information of tendon response at its transverse level during loading by a reduction of its CSA. The findings further add that those changes were gender-specific, with females demonstrating a higher AT-CSA deformation and compliance compared to males. As the incidence of AT injuries is higher in males compared to females, higher AT-CSA compliance might play a protective role towards stress-related injuries, possibly indicating higher adaptability to loading. Thus, to better understand tendon CSA behaviour and possible implications in performance and injury, its assessment should also be evaluated under functional tasks and between different populations where pathologies are present.

\section{Conflict of Interest}

The authors declare that they have no conflict of interest.

\section{References}

[1] Arya S, Kulig K. Tendinopathy alters mechanical and material properties of the Achilles tendon. J Appl Physiol 2010; 108: 670-675

[2] Bailey AJ, Paul RG, Knott L. Mechanisms of maturation and ageing of collagen. Mech Ageing Dev 1998; 106: 1-56

[3] Bryant AL, Clark RA, Bartold S, Murphy A, Bennell KL, Hohmann E, Marshall-Gradisnik S, Payne C, Crossley KM. Effects of estrogen on the mechanical behavior of the human Achilles tendon in vivo. J Appl Physiol 2008; 105: 1035-1043

[4] Burgess KE, Graham-Smith P, Pearson SJ. Effect of acute tensile loading on gender-specific tendon structural and mechanical properties. J Orthop Res 2009; 27: 510-516

[5] Carroll CC, Dickinson JM, Haus JM, Lee GA, Hollon C], Aagaard P, Magnusson SP, Trappe TA. Influence of aging on the in vivo properties of human patellar tendon. J Appl Physiol 2008; 105: 1907-1915

[6] Clayton RA, Court-Brown CM. The epidemiology of musculoskeletal tendinous and ligamentous injuries. Injury 2008; 39: 1338-1344

[7] Cook JL, Purdam CR. Is tendon pathology a continuum? A pathology model to explain the clinical presentation of load-induced tendinopathy. Br J Sports Med 2009; 43: 409-416

[8] Finni T, Komi PV, Lukkariniemi J. Achilles tendon loading during walking: application of a novel optic fiber technique. Eur J Appl Physiol Occup Physiol 1998; 77: 289-291

[9] Foure A, Cornu C, McNair PJ, Nordez A. Gender differences in both active and passive parts of the plantar flexors series elastic component stiffness and geometrical parameters of the muscle-tendon complex. J Orthop Res 2012; 5: 707-712

[10] Foure A, Nordez A, Cornu C. Plyometric training effects on Achilles tendon stiffness and dissipative properties. J Appl Physiol 2010; 109: 849-854

[11] Fukashiro S, Komi PV, Jarvinen M, Miyashita M. In vivo Achilles tendon loading during jumping in humans. Eur J Appl Physiol Occup Physiol 1995; 71: 453-458

[12] Giddings VL, Beaupre GS, Whalen RT, Carter DR. Calcaneal loading during walking and running. Med Sci Sports Exerc 2000; 32: 627-634

[13] Harriss DJ, Atkinson G. Ethical standards in sport and exercise science research: 2016 update. Int J Sports Med 2015; 36: 1121-1124

[14] Heinemeier KM, Kjaer M. In vivo investigation of tendon responses to mechanical loading. J Musculoskelet Neuronal Interact 2011; 11: 115-123

[15] Hess GW. Achilles tendon rupture: a review of etiology, population, anatomy, risk factors, and injury prevention. Foot Ankle Spec 2010; 3: 29-32

[16] Intziegianni K, Cassel M, Froehlich K, Engel T, Mayer F. Measuring achilles tendon length: a simple and reliable method. Sports Orthopaedics and Traumatology 2015; 31: 260-266 
[17] Intziegianni K, Cassel M, König N, Müller S, Fröhlich K, Mayer F. Ultrasonography for the assessment of the structural properties of the Achilles tendon in asymptomatic individuals: An intra-rater reproducibility study. Isokinetics and Exercise Science 2015; 23: 263-270

[18] Intziegianni K, Cassel M, Rauf S, White S, Rector M, Kaplick H, Wahmkow G, Kratzenstein S, Mayer F. Influence of age and pathology on Achilles tendon properties during a single-leg jump. Int J Sports Med 2016; 37: 973-978

[19] Kannus P. Structure of the tendon connective tissue. Scand J Med Sci Sports 2000; 10: 312-320

[20] Kannus P, Jozsa L. Histopathological changes preceding spontaneous rupture of a tendon. A controlled study of 891 patients. J Bone Joint Surg Am 1991; 73: 1507-1525

[21] Kastelic ], Palley I, Baer E. A structural mechanical model for tendon crimping. J Biomech 1980; 13: 887-893

[22] Kubo K, Kanehisa H, Fukunaga T. Gender differences in the viscoelastic properties of tendon structures. Eur J Appl Physiol 2003; 88: 520-526

[23] Maffulli N, Waterston SW, Squair ], Reaper ], Douglas AS. Changing incidence of Achilles tendon rupture in Scotland: a 15-year study. Clin J Sport Med 1999; 9: 157-160

[24] Maffulli N, Wong J. Rupture of the Achilles and patellar tendons. Clin Sports Med 2003; 22: 761-776

[25] Magnusson SP, Kjaer M. Region-specific differences in Achilles tendon cross-sectional area in runners and non-runners. Eur J Appl Physiol 2003; 90: 549-553

[26] Miller BF, Hansen M, Olesen JL, Schwarz P, Babraj JA, Smith K, Rennie MJ, Kjaer M. Tendon collagen synthesis at rest and after exercise in women. J Appl Physiol (1985) 2007; 102: 541-546

[27] Muraoka T, Muramatsu T, Fukunaga T, Kanehisa H. Geometric and elastic properties of in vivo human Achilles tendon in young adults. Cells Tissues Organs 2004; 178: 197-203

[28] O'Brien TD, Reeves ND, Baltzopoulos V, Jones DA, Maganaris CN. Mechanical properties of the patellar tendon in adults and children. J Biomech 2010; 43: 1190-1195
[29] Onambele GN, Burgess K, Pearson S]. Gender-specific in vivo measurement of the structural and mechanical properties of the human patellar tendon. J Orthop Res 2007; 25: 1635-1642

[30] Pang BS, Ying M. Sonographic measurement of achilles tendons in asymptomatic subjects: variation with age, body height, and dominance of ankle. J Ultrasound Med 2006; 25: 1291-1296

[31] Pearson SJ, Onambele GL. Computation methods affect the reported values of in vivo human tendon stiffness. J Mech Behav Biomed Mater 2012; 5: 291-297

[32] Portney LG, Watkins MP. Foundations of Clinical Research: Applications to Practice. Pearson/Prentice Hall; 2009

[33] Reed C, lozzo R. The role of decorin in collagen fibrillogenesis and skin homeostasis. Glycoconjugate J 2003; 19: 249-255

[34] Reeves ND, Maganaris CN, Narici MV. Effect of strength training on human patella tendon mechanical properties of older individuals. J Physiol 2003; 548: 971-981

[35] Riley G. The pathogenesis of tendinopathy. A molecular perspective. Rheumatology (Oxford) 2004; 43: 131-142

[36] Rosager S, Aagaard P, Dyhre-Poulsen P, Neergaard K, Kjaer M, Magnusson SP. Load-displacement properties of the human triceps surae aponeurosis and tendon in runners and non-runners. Scand J Med Sci Sports 2002; 12: 90-98

[37] Vosseller JT, Ellis SJ, Levine DS, Kennedy JG, Elliott AJ, Deland JT, Roberts MM, O'Malley MJ. Achilles tendon rupture in women. Foot Ankle Int 2013; 34: 49-53

[38] Wang JH. Mechanobiology of tendon. J Biomech 2006; 39: 1563-1582

[39] Waugh CM, Blazevich A], Fath F, Korff T. Age-related changes in mechanical properties of the Achilles tendon. J Anat 2012; 220: 144-155

[40] Westh E, Kongsgaard M, Bojsen-Moller ], Aagaard P, Hansen M, Kjaer $M$, Magnusson SP. Effect of habitual exercise on the structural and mechanical properties of human tendon, in vivo, in men and women. Scand J Med Sci Sports 2008; 18: 23-30 